\title{
Plastic Yielding as a Phase Transition
}

A statistical mechanical theory of forest hardening is developed in which yielding arises

M. Ortiz

Graduate Aeronautical Laboratories California Institute of Technology, Pasadena, CA 91125 Mem. ASME as a phase transition. For simplicity, we consider the case of a single dislocation loop moving on a slip plane through randomly distributed forest dislocations, which we treat as point obstacles. The occurrence of slip at the sites occupied by these obstacles is assumed to require the expenditure of a certain amount of work commensurate with the strength of the obstacle. The case of obstacles of infinite strength is treated in detail. We show that the behavior of the dislocation loop as it sweeps the slip plane under the action of a resolved shear stress is identical to that of a lattice gas, or, equivalently, to that of the two-dimensional spin- $\frac{1}{2}$ Ising model. In particular, there exists a critical temperature $T_{c}$ below which the system exhibits a yield point, i.e., the slip strain increases sharply when the applied resolved shear stress attains a critical value. Above the critical temperature the yield point disappears and the slip strain depends continuously on the applied stress. The critical exponents, which describe the behavior of the system near the critical temperature, coincide with those of the two-dimensional spin- $\frac{1}{2}$ Ising model.

\section{Introduction}

The aim of this paper is to show that yielding in crystals may be understood as a phase transition, and to establish conceptual links between theories of crystalline slip and the theory of critical phenomena.

A phase transition occurs in a system when there is a singularity in its free energy or one of its derivatives. Phase transitions manifest themselves as sharp changes in the properties of the system. Examples of phase transitions are the evaporation of a liquid into gas, the transition from a normal conductor to a superconductor, or from paramagnet to ferromagnet. The theory of critical phenomena is a well-developed discipline which delves on the commonalities in the behavior of seemingly disparate physical systems near their critical points. The theory has been successfully applied to a wide variety of systems and has led to the identification of universality classes obeying well-defined scaling laws (see, e.g., Stanley (1971), Binney et al. (1992), and Chaikin and Lubensky (1995)).

By contrast, the full potential of the theory of critical phenomena as regards the formulation of macroscopic constitutive theories for solids, and particularly to the understanding of crystal plasticity, is far from realized at present. The classical KTHNY theory on the statistical mechanics of ensembles of linear elastic dislocations in crystals (Kosterlitz and Thouless, 1972, 1973; Nelson and Halperin, 1979; Young, 1979; Chaikin and Lubensky, 1995) was mainly intended as a theory of defect-mediated melting and did not address issues related to the macroscopic plasticity of crystals. In particular, the KTHNY model of a dislocated crystal, which may be understood as a vectorial extension of the Coulomb gas model (Kosterlitz and Thouless, 1972, 1973), is not appropriate for the study of yielding, plastic flow, and hardening of crystals, as these phenomena are macroscopic manifestations of the motion of dislocations and their interaction with obstacles. More recently, Chrzan and Mills $(1993,1994)$ have argued for a connection between rates of hardening and critical exponents in $\mathrm{Ll}_{2}$ intermetallic compounds, and for the kind of scale invariance which accompa-

Contributed by the Applied Mechanics Division of The AmERICAN SOCIETy OF Mechanical Engineers for publication in the ASME Journal of Applied Mechanics. Discussion on the paper should be addressed to the Technical Editor, Professor Lewis T. Wheeler, Department of Mechanical Engineering, University of Houston, Houston, TX 77204-4792, and will be accepted until four months after final publication of the paper itself in the ASME Journal of APPLIED Mechanics.

Manuscript received by the ASME Applied Mechanics Division, Aug. 14, 1998 final revision, Nov. 20, 1998. Associate Technical Editor: L. T. Wheeler. nies criticality in the dislocation pinning-depinning transition in those materials. Chrzan and Mills pioneering work provides compelling evidence for a link between plastic yielding and criticality.

The work presented in this paper endeavors to demonstrate this link for the classical forest-hardening mechanism (e.g., Kovács, 1967; Kovács and Zsoldos, 1973; Cuitiño and Ortiz, 1992). In the forest-dislocation theory of hardening, the motion of dislocations, which are the agents of plastic deformation in crystals, is impeded by secondary - or "forest"- dislocations piercing through the slip plane. As the moving and forest dislocations intersect, they form junctions of varying strengths which may be idealized as point obstacles. The strength of some of these obstacles has recently been computed by Baskes et al. (1997) and Phillips and Shenoy (1998) using atomistic models. Moving dislocations are pinned down by the forest dislocations and require a certain elevation of the applied resolved shear stress in order to bow out and bypass the pinning obstacles. The net effect of this mechanism is a steady increase in the critical resolved shear stress required for macroscopic slip to operate, a phenomenon known as "hardening."

The simplest analytical treatments of the forest-hardening mechanism are based on a line-tension approximation. Thus, in these approaches long-range interactions between dislocation are entirely neglected. Despite its apparent coarseness, this approximation may be closer to reality than other formulations which painstakingly account for the interaction energy between dislocations, but fail to account for the formation of low-energy microstructures. Begin by recalling that the net Burgers vector-or net "charge" - of a dislocation ensemble must be zero. For instance, in multipolar arrangements this condition implies that there is an equal number of positive and negative dislocations in the ensemble. In addition, dislocations often tend to attain low-energy configurations (Hansen and Kuhlmann-Wilsdorff, 1986; KuhlmannWilsdorf, 1989). These are arrangements in which the long-range stress field of the dislocations vanishes. Roughly speaking, this is accomplished by surrounding each dislocation segment with segments of the opposite sign, i.e., by screening the segment; or by arranging dislocations as low-angle grain boundaries, dipolar walls, and other configurations for which the attendant plastic strains are compatible (Ortiz and Repetto, 1998). Under these circumstances, the remaining energy of the dislocations, or "selfenergy," is proportional to the dislocation length, as presumed in the line-tension approximation.

One of the most successful analytical treatments of the foresthardening mechanism was advanced by Kocks (1964) (see also 
Ortiz and Popov (1982) and Cuitiño and Ortiz (1992)), and is sometimes referred to as Kock's percolation model. In this approach, the applied resolved shear stress is equilibrated by the line tension of the dislocations. The critical stress $\tau_{c}$ required for a dislocation segment to bypass a pair of pinning point obstacles is proportional to the line tension and inversely proportional to the distance between the obstacles. If the point obstacles are assumed to be randomly distributed over the slip plane, it follows that $\tau_{c}$ itself is a random variable with a well-characterized probability density function $f\left(\tau_{c}\right)$ (Grosskreutz and Mughrabi, 1975; Mughrabi, 1975). As the resolved shear stress is raised from $\tau$ to $\tau+\Delta \tau$, all dislocation segments with $\tau_{c}$ in the interval $[\tau, \tau+\Delta \tau]$ become unstable and effect random flights. The length of these flights, and the attendant slip-strain increment, was estimated by Kocks (1964) by imagining that the dislocation segments move over a onedimensional "obstacle course" consisting of obstacles of random heights distributed as $f\left(\tau_{c}\right)$. In particular, the segments arrest when they reach obstacles of strength greater than $\tau+\Delta \tau$.

It is clear that the one-dimensional character of this analysis represents a sweeping simplification as regards the geometry and topology of moving dislocation loops. For instance, an expanding dislocation loop may become pinched, with the result that the number of connected components of the loop is increased by one. Conversely, loops can shrink to a point or be left behind as debris surrounding point obstacles, or Orowan loops. Evidently, these intricacies are not accounted for in one-dimensional renditions of the forest-hardening mechanism. More detailed analyses of foresthardening have invariably been based on numerical methods (Foreman and Makin, 1966, 1967). Despite these limitations, Kock's percolation model, or subsequent extensions there of (Ortiz and Popov, 1982; Cuitiño and Ortiz, 1992; Kocks et al., 1991) has provided an effective basis for describing the hardening of materials such as fcc metals (Cuitiño and Ortiz, 1993; Cuitiño, 1996), $\mathrm{L1}_{2}$ intermetallic compounds (Cuitiño and Ortiz, 1993), and others.

The theory developed in this paper overcomes the topological restrictions inherent to one-dimensional treatments of the foresthardening mechanism, and establishes a clear link between yielding and criticality. In order to establish this link in the simplest possible terms, we restrict our attention to the motion of a single dislocation loop through a slip plane containing a random array of point obstacles. As discussed above, we assume that the dislocations are well screened and hence their energy is ostensibly proportional to their length. In the spirit of level-set methods, the dislocation loop is described by a scalar field $\xi$ which takes the value of 1 inside the loop and 0 outside the loop. The transition between these two extreme values occurs over the dislocation line. In this manner, no restrictions are placed on the evolving geometry and topology of the loop. In order to simplify the analysis, however, we discretize the field $\xi$ on a square lattice spanning the slip plane. The motion of the loop is impeded by forest dislocations piercing the slip plane, which we treat as point obstacles. The occurrence of slip at the sites occupied by these obstacles is assumed to require the expenditure of a certain amount of work commensurate with the strength of the obstacle.

The equilibrium properties of the dislocation loop/obstacle system may be described within the framework of Gibbsian statistical mechanics. We show that, once the effect of the point obstacles is taken into account, the effective Hamiltonian of the system is identical to that of a lattice gas, or, by a simple change of variables, to the two-dimensional spin- $\frac{1}{2}$ Ising model (see, e.g., Yeomans (1992)). In the parlance of the theory of critical phenomena, crystals whose plasticity is well described by the forest-hardening mechanism belong to the universality class of the two-dimensional spin- $-\frac{1}{2}$ Ising model. In particular, the behavior and scaling properties of all such crystals should be identical near the critical point, and be described by a few material-independent critical exponents. For zero applied field, the spin- $\frac{1}{2}$ Ising model was solved by Onsager (Baxter, 1982), and to date furnishes one of the rare few examples of nontrivial model systems which can be solved exactly.
In particular, the critical exponents are known exactly for the two-dimensional spin- $\frac{1}{2}$ Ising model and, by extension, for the forest-hardening model developed in this paper.

Systems which can be described by the two-dimensional spin- $\frac{1}{2}$ Ising model exhibit a phase transition at a critical temperature $T_{c}$. In the particular case of the dislocation loop/obstacle system, below the critical temperature this transition is signalled by a sudden increase in the slip strain. For sufficiently low temperatures, the system jumps from a state characterized by a slip strain close to zero to a state characterized by generalized slip over most of the slip plane. The critical resolved shear stress at which yielding occurs follows from the theory as a function of temperature, the obstacle density and material constants. The size of the slip-strain jump at yielding decreases to zero as the critical temperature is approached from below, and disappears altogether above the critical temperature. In this latter regime, therefore, the slip strain depends continuously on the applied resolved shear stress and no yield point is in evidence. However, examination of model materials, such as copper, suggests that $T_{c}$ is close to the melting temperature. Therefore, yielding in these materials is predicted to persist up to very high temperatures, in keeping with observation.

Unfortunately, no exact solution is presently known for the two-dimensional spin- $-\frac{1}{2}$ Ising model in the presence of an applied field. A simple approximate solution may be obtained by recourse to mean-field theory. Mean-field theory reveals compelling geometrical insights into the equilibrium properties of the system. In addition, it suggests the rudiments of a kinetic theory which accounts for hysteresis. This rudimentary kinetics is in analogy to that which is envisioned in theories of hysteresis proposed for wires or bars which undergo martensitic transformations (Abeyaratne and Knowles, 1988). It should be emphasized, however, that the resulting kinetic model represents an extension of equilibrium statistical mechanics, in as much as it requires additional assumptions regarding the accessibility of states in phase space. These restrictions on accessibility clearly violate ergodicity and place the model outside the realm of equilibrium statistical mechanics.

The paper is organized as follows. In Section 2 the Hamiltonian of the dislocation loop/obstacle system is formulated. This Hamiltonian includes terms which account for the self-energy of the dislocations, computed from the line-tension approximation, the applied resolved shear stress, and the point obstacles introduced in the slip plane by forest dislocations. In particular, a chemical potential is introduced as a device for controlling the density of point obstacles. In Section 3 the equilibrium properties of the system are established within the framework of Gibbsian statistical mechanics. These developments lead to he computation of the internal and free energies of the system, and the attendant relations between slip strain, applied resolved shear stress, temperature, and obstacle density. In Section 4, the nature of the phase transition and its relation to yielding is uncovered with the aid of mean-field theory. In Section 5, a simple kinetic theory is developed which accounts for hysteresis and plastic dissipation. Possible extensions of the theory and suggestions for further work are discussed in Section 6

\section{Forest Hardening}

The aim next is to formulate a simple model of forest-hardening mechanism which captures the essential physics of the mechanism while lending itself to a full analytical treatment within the framework of equilibrium statistical mechanics. In the interest of simplicity, we focus on the motion of a single dislocation loop through a random array of point obstacles corresponding to the intersections between the slip plane and forest dislocations.

We begin by discretizing the slip plane into a square lattice of parameter $a$ (Fig. 1). In order to avoid infinite sums, we consider a square subdomain containing $N$ sites, e.g., with periodic boundary conditions enforced on its boundary. The thermodynamic limit 


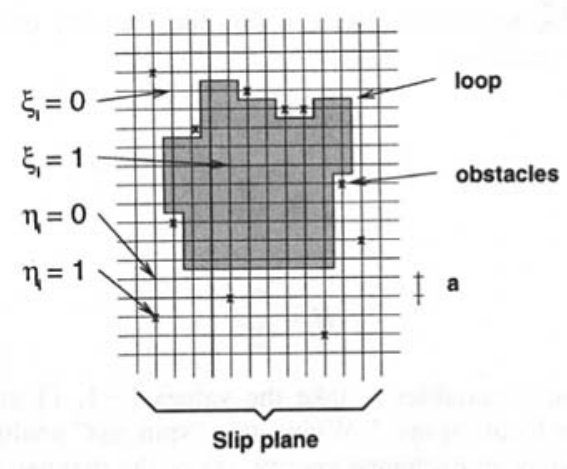

Fig. 1 Schematlc of the dislocation/obstacle system

of interest may then be attained by letting $N \rightarrow \infty$ and is independent of the precise nature of the boundary conditions. Each point $i=1, \ldots, N$ in the lattice is assigned a variable $\xi_{i}$ of value 1 if the point is within the dislocation loop and 0 if it is without (Fig. 1). Thus $\xi_{i}$ is the characteristic lattice function of the area covered by the dislocation loop. The lattice function $\xi_{i}$ may also be regarded as a two-state field describing the configuration of the loop. The dislocation line may be identified with the collection of segments which join lattice sites in different states, i.e., unslipped lattice sites, $\xi_{l}=0$, with slipped lattice sites, $\xi_{i}=1$.

We shall assume that the dislocation loop is well screened, so that the main contribution to its energy arises from its self-energy. For simplicity, we shall assume that the self-energy $\Gamma$ per unit dislocation length, or line tension, is isotropic, i.e., does not depend on the local orientation of the dislocation segments. An estimate for the line tension is (e.g., Hirth and Lothe (1968))

$$
\Gamma=C G b^{2}
$$

where $G$ is the elastic shear modulus, $b$ is the Burgers vector length, and $C$ is a constant of order unity. It should be noted that $G$ is strongly temperature-dependent in general and may be expected to reduce to zero at the melting temperature.

With these assumptions, the self-energy of the dislocation loop represented by the field $\xi_{i}$ is

$$
E=\sum_{\langle i, j\rangle} \Gamma a\left(\xi_{i}-\xi_{j}\right)^{2}
$$

where the sum is restricted to nearest-neighbor pairs, and $\Gamma a$ may be regarded as an "exchange" energy. Indeed, for a straight dislocation aligned with the lattice, it is readily verified that Eq. (2) gives an energy per unit length equal to $\Gamma$. If, in addition, the slip plane is under the action of a resolved shear stress $\tau$, then the total energy becomes

$$
E=\sum_{\langle i, j\rangle} \Gamma a\left(\xi_{i}-\xi_{j}\right)^{2}-\tau b a^{2} \sum_{i=1}^{N} \xi_{i}
$$

where $b$ is the Burgers vector length and $\tau b a^{2}$ plays the role of an applied field. The energy (2) is direct analogy to that of a lattice gas (Stanley, 1971; Yeomans, 1992).

Next we wish to model the effect of forest dislocations piercing the slip plane and hindering the motion of the loop. We shall restrict the intersection points to coincide with the lattice sites. A pinned mobile dislocation is assumed to drag the point obstacle when the force exerted by the dislocation on the obstacle attains a critical value $f$, which may be regarded as the strength of the obstacle. As the dislocation drags the obstacle one lattice distance, it does work in the amount fa. Heidenreich and Shockley (Nabarro, 1967) were the first to make a quantitative estimate of the work required to cause two dislocations to cross. They showed that, when two dislocations cut, each acquires a jog with an energy which they estimated as $G b^{3}$. Nabarro (1967) later refined this estimate to $G b^{3} / 4 \pi$ for each jog or $G b^{3} / 2 \pi$ in all. Using Nabarro's formula with $a=b$ the strength of a forest obstacle is computed to be

$$
f=G b^{2} / 2 \pi .
$$

Since the strength of the obstacles is proportional to the shear modulus, it may be expected to reduce to zero at the melting temperature, which accounts for the observed thermal softening of crystals. For simplicity, we restrict our attention to the case of one species of point obstacles of uniform strength. Under these assumptions, the total energy of the system becomes

$$
E=\sum_{\langle i, j\rangle} \Gamma a\left(\xi_{i}-\xi_{j}\right)^{2}-\tau b a^{2} \sum_{i=1}^{N} \xi_{i}+f a \sum_{i=1}^{N} \xi_{i} \eta_{i}
$$

where $\eta_{i}$ is 1 if site $i$ contains a point obstacle, and 0 otherwise, Fig. 1. The four possible states at a site $i$ are, therefore,

$1 \xi_{i}=0, \eta_{i}=0$ : the site has not slipped and is not occupied by a point obstacle; the work done against obstacles is zero.

$2 \xi_{i}=1, \eta_{i}=0$ : the site has slipped and is not occupied by a point obstacle; the work done against obstacles is zero.

$3 \xi_{i}=0, \eta_{i}=1$ : the site has not slipped and is occupied by a point obstacle; the work done against obstacles is zero.

$4 \xi_{i}=1, \eta_{i}=1$ : the site has slipped and is occupied by a point obstacle; the work done against the obstacle is $f a$.

Evidently, the effect of the last term in (5) is to introduce an energetic barrier to slip across obstacles. This barrier effectively pins the dislocations and forces the dislocation loops to bow out between obstacles, which is the desired effect. cles

Finally, we wish to have control on the number of point obsta-

$$
n=\sum_{i=1}^{N} \eta_{i}
$$

populating the slip plane. A conventional means of accomplishing this control is to introduce a chemical potential $\mu$, whereupon the total energy becomes

$$
\begin{aligned}
E=\sum_{\langle i, j\rangle} \Gamma a\left(\xi_{i}-\xi_{j}\right)^{2}-\tau b a^{2} \sum_{i=1}^{N} & \xi_{i} \\
& \quad+f a \sum_{i=1}^{N} \xi_{i} \eta_{i}-\mu \sum_{i=1}^{N} \eta_{i} .
\end{aligned}
$$

This energy constitutes the basis for all subsequent developments, and is the centerpiece of the present theory.

\section{Equilibrium Thermodynamic Behavior}

We proceed to explore the equilibrium properties of the dislocation loop/obstacle model introduced in the foregoing. According to the fundamental principle of equilibrium statistical mechanics (Feynman, 1972), the probability that a system in equilibrium be in a state $\boldsymbol{\xi}=\left\{\xi_{1}, \ldots, \xi_{N}\right\}$ and $\boldsymbol{\eta}=\left\{\eta_{1}, \ldots, \eta_{N}\right\}$ is

$$
p(\boldsymbol{\xi}, \boldsymbol{\eta})=\frac{1}{Z} e^{-\beta E(\xi, \eta)}
$$

where

$$
\beta=\frac{1}{k T}
$$

$k$ is Boltzmann's constant, $T$ is the absolute temperature, and 


$$
Z=\sum_{\xi_{1} \in\{0,1\}} \cdots \sum_{\xi_{N} \in\{0,1\}} \sum_{\eta_{1} \in\{0,1\}} \cdots \sum_{\eta N \in\{0,1\}} e^{-\beta E(\xi, \eta)}
$$

is the partition function. The thermodynamic properties of interest follow directly from $Z$. For instance, the internal energy per unit volume is

$$
U=\frac{1}{a^{2} l} \lim _{N \rightarrow \infty} \frac{1}{N}\left\{-\frac{\partial \log Z}{\partial \beta}\right\}
$$

whereas the free energy per unit volume is

$$
F=\frac{1}{a^{2} l} \lim _{N \rightarrow \infty} \frac{1}{N}\left\{-\frac{\log Z}{\beta}\right\} .
$$

Here $l$ is the distance between slip planes. The expected number of point obstacles per unit area follows as

$$
c=\frac{1}{a^{2}} \lim _{N \rightarrow \infty} \frac{1}{N}\left\langle\sum_{i=1}^{N} \eta_{i}\right\rangle=-l \frac{\partial F}{\partial \mu}
$$

and the slip strain is

$$
\gamma=\frac{b}{l} \lim _{N \rightarrow \infty} \frac{1}{N}\left\langle\sum_{i=1}^{N} \xi_{i}\right\rangle=-\frac{\partial F}{\partial \tau} .
$$

In addition, the mobile dislocation length $\rho$ on the slip plane per unit volume of the crystal is given by

$$
\rho=\frac{1}{a l} \lim _{N \rightarrow \infty} \frac{1}{N}\left\langle\sum_{\langle i, j\rangle}\left(\xi_{i}-\xi_{j}\right)^{2}\right\rangle=\frac{\partial F}{\partial \Gamma} .
$$

As may be seen, this dislocation density is closely related to the two-point correlation function of the slip distribution.

The partition function corresponding to energy (7) may be evaluated as follows. The sum over the obstacle occupancy field $\boldsymbol{\eta}$ is trivial and gives

$$
Z=\sum_{\xi_{1} \in\{0,1\}} \cdots \sum_{\xi_{N} \in\{0,1\}} e^{-\beta E(\xi)} \prod_{i=1}^{N}\left[1+e^{-\beta\left(f a \xi_{i}-\mu\right)}\right]
$$

where $E(\xi)$ is given by (3). Alternatively, (16) may be recast in the form

$$
\begin{aligned}
Z=\sum_{\xi_{1} \in\{0,1\}} \cdots \sum_{\xi_{N} \in\{0,1\}} \exp \{-\beta[E(\boldsymbol{\xi}) \\
\left.\left.\quad-\frac{1}{\beta} \sum_{i=1}^{N} \log \left(1+e^{-\beta\left(f a \xi_{i}-\mu\right)}\right)\right]\right\} .
\end{aligned}
$$

But, since $\xi_{i}$ takes the values 0 and 1 only, we can write

$$
\begin{aligned}
\log \left(1+e^{-\beta\left(f a \xi_{i}-\mu\right)}\right) & =\log \left(1+e^{-\beta(f a-\mu)}\right) \xi_{i} \\
+ & \log \left(1+e^{\beta \mu}\right)\left(1-\xi_{i}\right), \quad \xi_{i} \in\{0,1\} .
\end{aligned}
$$

Inserting this identity into (17) gives

$$
\begin{aligned}
Z=\left(1+e^{\beta \mu}\right)^{N} & \sum_{\xi \in\{0.1\}} \cdots \\
& \sum_{\xi_{N} \in\{0,1\}} \exp \left\{-\beta\left[\sum_{\langle i, j\rangle} \Gamma a\left(\xi_{i}-\xi_{j}\right)^{2}-\epsilon \sum_{i=1}^{N} \xi_{i}\right]\right\}
\end{aligned}
$$

where

$$
\epsilon=\tau b a^{2}+\frac{1}{\beta} \log \left(\frac{1+e^{-\beta(f a-\mu)}}{1+e^{\beta \mu}}\right)
$$

It is possible to reduce (19) to a familiar form by the following change of variables:

$$
\begin{gathered}
s_{i}=2 \xi_{i}-1 \\
J=\frac{\Gamma a}{2} \\
H=\frac{\epsilon}{2} .
\end{gathered}
$$

The new state variables $s_{i}$ take the values $\{-1,1\}$ and may be regarded as local "spins"." Within this "spin gas" analogy, $J$ may be regarded as an exchange energy, $\langle\xi\rangle$ as the magnetization, and $H$ as an applied field. Inserting these definitions into (19) gives

$$
Z=\left(1+e^{\beta \mu}\right)^{N} e^{-\beta(J-H) N} Z^{\text {ising }}(K, h)
$$

where

$$
\begin{aligned}
& K=\beta J \\
& h=\beta H
\end{aligned}
$$

and

$$
\begin{gathered}
Z^{\text {ising }}=\sum_{s_{i}= \pm 1} \cdots \sum_{s_{N}= \pm 1} e^{-\beta E^{\text {ising }(\mathbf{s})}} \\
E^{\text {ising }}(\mathbf{s})=-K \sum_{\langle i, j\rangle} s_{i} s_{j}-h \sum_{i=1}^{N} s_{i}
\end{gathered}
$$

are the partition function and energy of the two-dimensional spin- $\frac{1}{2}$ Ising model. Substitution of (24) into (12) gives the free-energy density of the loop/obstacle system as

$F=\frac{1}{a^{2} l}\left\{-\frac{1}{\beta} \log \left(1+e^{\beta \mu}\right)+J-H\right\}+F^{\text {ising }}(K, h)$.

Thus, the equilibrium properties of the loop/obstacle system are closely related to those of the two-dimensional spin- $-\frac{1}{2}$ Ising model, which are well understood at present (e.g., Baxter (1982)).

Subsequent calculations may be simplified by the introduction of the following dimensionless variables:

$$
\begin{gathered}
t=\beta \tau b a^{2} \\
m=\beta \mu \\
\varphi=\beta F a^{2} l
\end{gathered}
$$

We also note the identities

$$
\begin{gathered}
\langle\xi\rangle=\lim _{N \rightarrow \infty} \frac{1}{N}\left\langle\sum_{i=1}^{N} \xi_{i}\right\rangle=\frac{\gamma}{\gamma^{\text {sat }}} \\
r \equiv \lim _{N \rightarrow \infty} \frac{1}{N}\left\langle\sum_{\langle i j\rangle}\left(\xi_{i}-\xi_{j}\right)^{2}\right\rangle=\frac{\rho}{\rho^{\text {sat }}} \\
\langle\eta\rangle=\lim _{N \rightarrow \infty} \frac{1}{N}\left\langle\sum_{i=1}^{N} \eta_{i}\right\rangle=\frac{c}{c^{\text {sat }}}
\end{gathered}
$$

which follow from (13), (14), and (15) with

$$
\begin{gathered}
\gamma^{\text {sat }}=\frac{b}{l} \\
\rho^{\text {sat }}=\frac{1}{a l}
\end{gathered}
$$




$$
c^{\text {sat }}=\frac{1}{a^{2}}
$$

Evidently, these are: the saturation slip strain which is attained when the loop sweeps the entire slip plane; the saturation dislocation density which is obtained, e.g., when the dislocation population consists of parallel straight dislocations at a distance $a$; and the saturation obstacle density, which is obtained when the forest dislocations intersect the slip plane through every lattice site. We also note that $\langle\xi\rangle$ plays the role of a normalized slip strain, $r$ the role of a normalized dislocation density, and $\langle\eta\rangle$ the role of a normalized obstacle density, all ranging from 0 to 1 . In terms of these variables (29) becomes

$$
\varphi=-\log \left(1+e^{m}\right)+K-h+\varphi^{\text {ising }}(K, h)
$$

with

$$
h=\frac{t}{2}-\frac{1}{2} \log \left(1+e^{m}\right)
$$

whereas (13), (14), and (15) simplify to

$$
\begin{gathered}
\langle\xi\rangle=-\frac{\partial \varphi}{\partial t} \\
r=\frac{1}{4} \frac{\partial \varphi}{\partial K} \\
\langle\eta\rangle=-\frac{\partial \varphi}{\partial m} .
\end{gathered}
$$

This last relation may be inverted to determine the chemical potential $m$ as a function of the obstacle density $\langle\eta\rangle$.

\section{Criticality and Yielding}

Criticality underlies and unifies a number of important physical phenomena such as the transitions from liquid to gas, from a normal conductor to a superconductor, and from paramagnetic to ferromagnetic behavior (e.g., Stanley (1971), Feynman (1972), Yeomans (1992), Binney et al. (1992), and Chaikin and Lubensky (1995)). As is well known, the phase diagram of the twodimensional spin $-\frac{1}{2}$ Ising model exhibits a phase transition (e.g., Yeomans (1992)). In the present theory, this phase transition provides a model for the yield phenomenon in crystals.

Unfortunately, the two-dimensional spin- $\frac{1}{2}$ Ising model with nonzero field has not been solved analytically. The numerical calculation of $F^{\text {ising }}$ offers no particularly difficulties and is extensively discussed in the literature (e.g., Binder, ed. $(1986,1987)$ and Koonin and Meredith (1990)). Here, however, in order to obtain explicit results we shall resort to a mean-field approximation (Yeomans, 1992; Chaikin and Lubensky, 1992). We begin by introducing an effective or mean-field energy of the form

$$
E_{0}=-H_{0} \sum_{i=1}^{N} s_{i}
$$

and we seek to optimize the value of the effective field $H_{0}$. To this end, we recall Bogoliubov's inequality

$$
F \leq F_{0}+\lim _{N \rightarrow \infty} \frac{1}{N} \frac{1}{a^{2} l}\left\langle E-E_{0}\right\rangle_{0}
$$

where $F$ is the free energy per unit volume, Eq. (12), corresponding to the original energy $E$, the average $\langle\cdot\rangle_{0}$ is taken with respect to the mean-field probability,

$$
p_{0}(\mathbf{s})=\frac{1}{Z_{0}} e^{-\beta E_{0}(\mathbf{s})}
$$

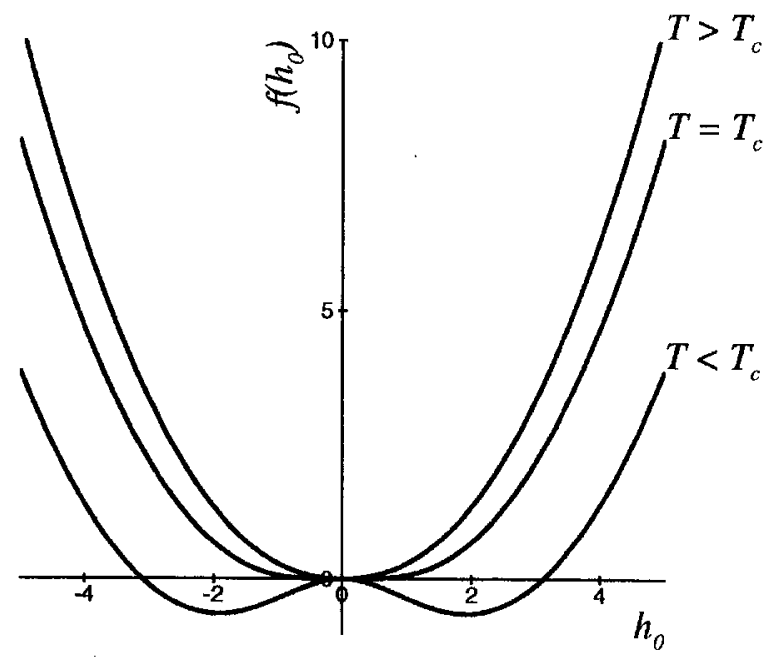

Fig. 2 Bogoliubov's function $f\left(h_{0}\right)$ shown in the zero applied fleld case, $h=0$

$$
Z_{0}=\sum_{s_{1}= \pm 1} \cdots \sum_{s_{N}= \pm 1} e^{-\beta E_{0}(s)}
$$

and $F_{0}$ is the free energy per unit volume corresponding to the mean-field energy $E_{0}$. Inequality (45) holds for any choice of energy $E_{0}$ (e.g., Feynman (1972)), with the equality sign attained for $E_{0}=E$. Inserting (28) and (44) and using normalization (32) and (26), the bound in (45) becomes

$$
f\left(h_{0}\right)=\frac{1}{2} h_{0}^{2}-\kappa \log \left(\cosh h_{0}\right)-h h_{0} .
$$

The optimum value of the effective field $h_{0}$ is identified with the absolute minimizer of $f\left(h_{0}\right)$. The resulting mean-field free energy for the two-dimensional spin- $\frac{1}{2}$ Ising model with nonzero applied field is (cf. Yeomans (1992)):

$$
\varphi^{\text {ising }} \approx \min _{h_{0}} f\left(h_{0}\right)=-\log \left(2 \cosh h_{0}\right)+\frac{1}{2} K z \tanh ^{2} h_{0}
$$

where $z$ is the coordination number of the lattice, e.g., $z=4$ for the square lattice. A trite but straightforward calculation of (41), (42), and (43) using (49) gives

$$
\begin{gathered}
\langle\xi\rangle=\frac{1}{2}\left(1+\tanh h_{0}\right) \\
r=\frac{z}{8}\left(1-\tanh ^{2} h_{0}\right) \\
\langle\eta\rangle=\frac{1}{2}\left(1-\tanh h_{0}\right) \frac{e^{m}}{1+e^{m}} .
\end{gathered}
$$

Equations (50) and (51) yield the normalized shear strain and dislocation density, respectively, whereas (52) can be used to eliminate the chemical potential $m$ in favor of the obstacle density $\langle\eta\rangle$.

The function $f\left(h_{0}\right)$, Eq. (48), is shown in Fig. 2 for the zero applied field case, $h=0$. It is seen from this figure that $f\left(h_{0}\right)$ is convex in the supercritical regime $K<K_{c}$, where

$$
K_{c}=\frac{1}{z}
$$

defines a critical value of $K$. By contrast, in the subcritical regime, $K>K_{c}, f\left(h_{0}\right)$ is nonconvex and exhibits two minima, or wells, separated by a maximum. It is therefore expected that the behavior of the system will differ sharply in the two regimes, and that the condition $K=K_{c}$ signals the onset of a phase transition. The corresponding critical temperature is 


$$
T_{c}=K_{c}^{-1} \frac{J}{k}=K_{c}^{-1} \frac{\Gamma a}{2 k}=K_{c}^{-1} \frac{C G b^{2} a}{2 k}
$$

and, thus, the subcritical and supercritical regimes correspond to the temperature ranges $T<T_{c}$ and $T>T_{c}$, respectively.

As a check on the accuracy of the mean field approximation it may be noted that the exact critical point of the Ising model on a square lattice $(z=4)$ at zero applied field is $K_{c}=\frac{1}{2} \log (1+$ $\sqrt{2}$ ) $\approx 0.44069$, whereas the mean-field critical point is $K_{c}=\frac{1}{4}$, which is somewhat lower than the exact value. The mean-field critical exponents also differ from those obtained from an exact treatment of the Ising model. Despite these discrepancies, the mean-field theory does furnish a simple and analytically tractable model which exhibits a phase transition, and thus suffices to demonstrate the connection between criticality and yielding pursued here.

The extrema of the function $f\left(h_{0}\right)$, Eq. (48), are the solutions of the secular equation

$$
h=h_{0}-\kappa \tanh h_{0}
$$

Here and subsequently we write

$$
\kappa=K z=\frac{K}{K_{c}} .
$$

The solutions of Eq. (55) in turn correspond to the intersections of the function $h_{0}-\kappa \tanh h_{0}$ with the horizontal line of height $h$ (Figs. $3(a)$ and $3(b)$ ). It is clear from these figures that $f\left(h_{0}\right)$ has a single minimum $h_{0}$ in the supercritical case $T>T_{c}$ (Fig. 3(a), point $A$ ), and that $h_{0}$ depends continuously on $h$. In view of (50), (51), (52), and (40) it follows that the slip strain and the dislocation density are continuous functions of the applied resolved shear stress and no yielding occurs in the supercritical regime.

The subcritical or low-temperature equilibrium behavior of the loop/obstacle system is more eventful and may be characterized as follows. The function $h_{0}-\kappa \tanh h_{0}$ attains the maximum

$$
h_{c}=\operatorname{acosh} \sqrt{\kappa}-\sqrt{\kappa(\kappa-1)}
$$

at $h_{0}=\operatorname{acosh} \sqrt{\kappa}$. In the range $h<-h_{c}$, which by virtue of Eq. (40) corresponds to a low applied shear stress $t$, Eq. (55) has a unique solution $h_{0}$ (Fig. 3(b), point $A$ ). An increase in $h$, e.g., due to an elevation in the applied resolved shear stress $t$, results in a corresponding increase in $h_{0}$ and $\langle\xi\rangle$. In the range $-h_{c}<h<h_{c}$, the horizontal line at $h$ intersects the function $h_{0}-\kappa \tanh h_{0}$ at three points, Fig. $3(b)$, corresponding to three extrema of $f\left(h_{0}\right)$. Of these extrema, the central point is a maximum and the remaining points are minima. Of these two minima, the left point $h_{0}<0$ is the absolute minimizer in the range $h<0$ (Fig. $3(b)$, point $B$ ), and the right point $h_{0}>0$ is the absolute minimizer in the range $h>$ 0 (Fig. 3(b), point $C$ ). The point of exchange of stability is, therefore, $h=0$, at which point the effective field $h_{0}$ jumps discontinuously to a larger value. This jump is accompanied by a sudden increase in slip activity and may, therefore, be identified with the yield point of the system. In the range $h>h_{c}$, corresponding to a high applied shear stress $t$, Eq. (55) has again a unique solution $h_{0}$ (Fig. $3(b)$, point $D$ ).

It may be noted that, at zero temperature, the yield transition just described is in analogy to solid-solid phase transitions in bars and wires, and the applied field $h=0$ plays a role analogous to the Maxwell stress (Ericksen, 1975; James, 1979). This analogy will be exploited further in Section 5 with a view to formulating a simple kinetic theory which accounts for hysteresis and plastic dissipation.

The critical resolved shear stress corresponding to the yield point $h=0$ follows from Eq. (40) as

$$
t_{c}=\log \left(1+e^{m}\right)
$$

Equivalently, $t_{c}$ may be rewritten in terms of the obstacle density using (52), with the result

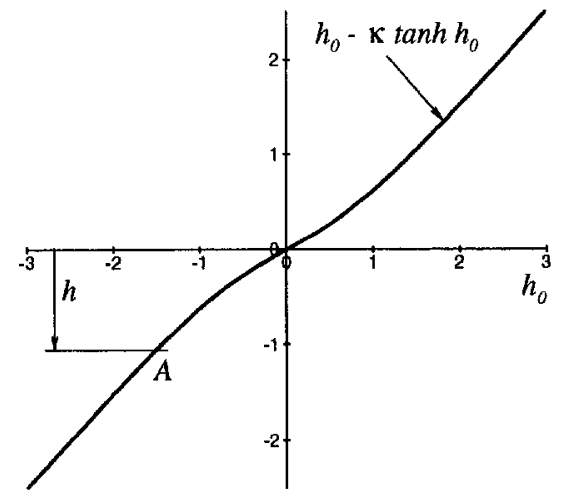

(a)

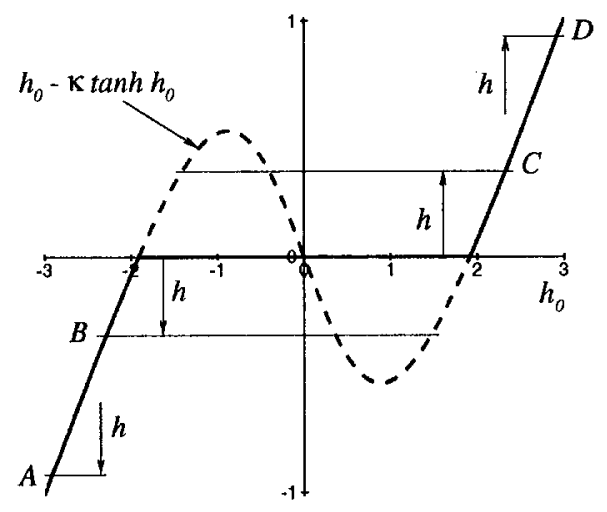

(b)

Fig. 3 Mean-field construction for the determInation of the effective fleld $h_{0}$ from the applled fleld $h$. (a) Supercritical case $k=\frac{1}{2} ;(b)$ subcritical case $\kappa=2$.

$$
t_{c}=-\log \left(1-\frac{2\langle\eta\rangle}{1+\tanh h_{0 c}}\right)
$$

where $h_{0 \mathrm{c}}$ is the positive root of the equation

$$
h_{0}-\kappa \tanh h_{0}=0 .
$$

It follows from this relation that the critical resolved shear stress vanishes in the absence of point obstacles, $\langle\eta\rangle=0$, and diverges to infinity at the "percolation limit"

$$
\langle\eta\rangle=\frac{1+\tanh h_{0 c}}{2} .
$$

As the critical temperature is approached from below, one has that $h_{0 c} \rightarrow 0$ and the percolation limit is attained when one-half of the lattice sites are occupied by obstacles. In the opposite extreme of zero temperature, the root $h_{0 c}$ diverges to infinity and the percolation limit is attained when all lattice sites are occupied by obstacles.

Figure 4 displays the equilibrium properties of the system in the subcritical regime $T<T_{c}$. The evolution of the slip strain $\langle\xi\rangle$ with applied resolved shear stress $t$ is shown in Fig. 4(a) for three values of the obstacle density $\langle\eta\rangle$. As expected, the critical resolved shear stress hardening rate increase with obstacle density. Figure $4(b)$ shows the dependence of the chemical potential $m$ on the slip 


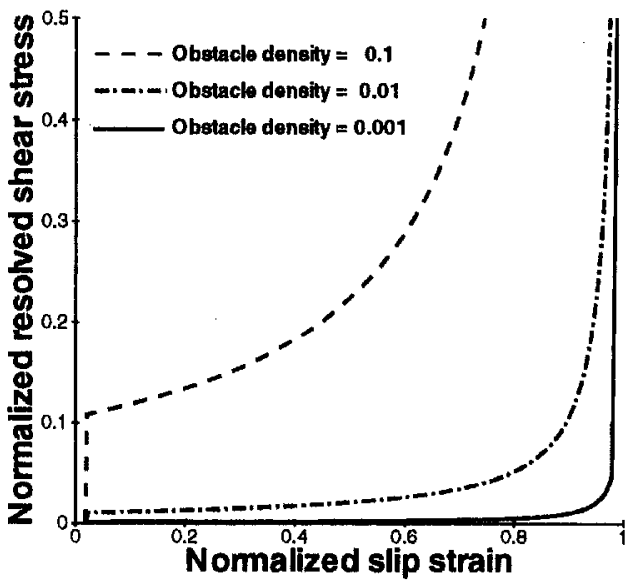

(a)

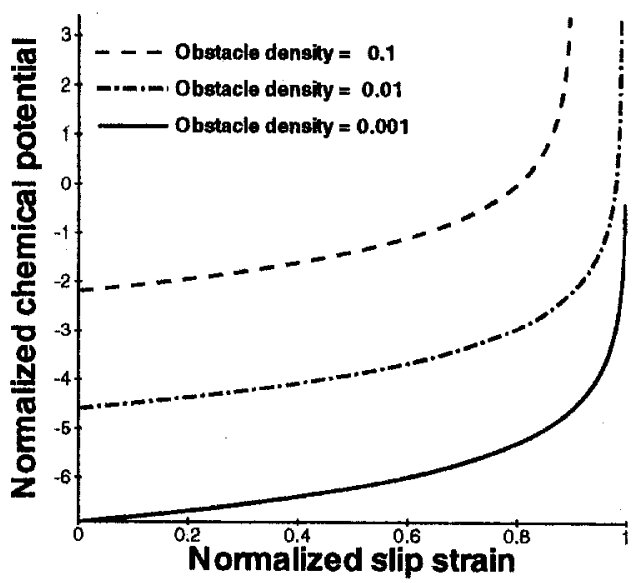

(b)

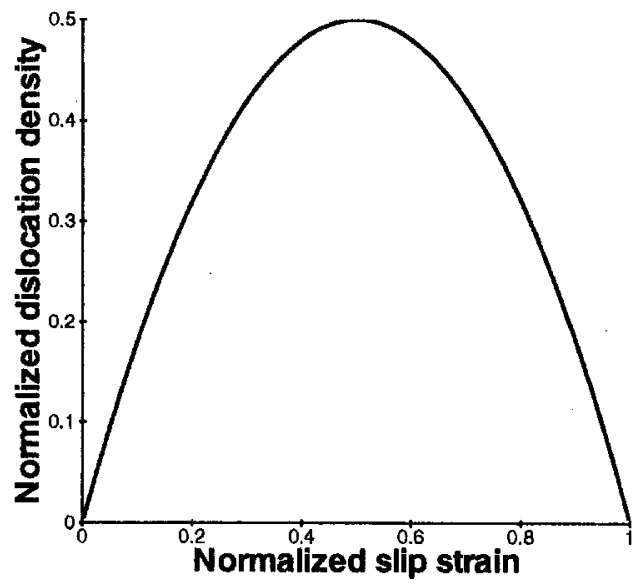

(c)

Fig. 4 (a) Normalized resolved shear stress $t=\beta \tau b a^{2} ;(b)$ normalized chemical potential $m=\beta \mu$; and $(c)$ normallzed dislocation density $r=\rho a^{2}$ versus the normalized slip strain $\langle\xi\rangle=\gamma /(b / l)$ for three values of the normalized obstacle density $\langle\eta\rangle=c a^{2}$ In the subcritical regime, $\kappa=2$

strain and obstacle density. It is seen from the figure that the chemical pontential is an increasing function of the obstacle density. It is also interesting to note how, as the slip strain and, consequently, the energy of interaction between the dislocation loop and the obstacles increases, it becomes necessary to compensate by raising the chemical potential in order to maintain the number of obstacles constant. The variation of the dislocation density with slip is also noteworthy (Fig. 4(c)). During the early stages of loading, the dislocation density $r$ is close to zero. In this stage, the mobile dislocation population consists of small loops covering a small area fraction of the slip plane. Physically, these loops emanate from dislocation sources, which we assume to be plentiful and easy to operate. In the neighborhood of the yield point, the mobile dislocation density explodes to accommodate the rapidly increasing slip strain, attains the maximum $z / 8$, and subsequently decreases monotonically to zero as dislocation dipoles annihilate. As the slip strain nears its saturation value, the dislocation population consists of small debris, or Orowan, loops surrounding the forest obstacles, slip having occurred everywhere outside the loops.

If follows from the preceding discussion that the critical point $T=T_{v}$ is marked by divergences in the zero-field specific heat

$$
C_{H}=\left(\frac{\partial U}{\partial T}\right)_{H}
$$

and the plastic compliance

$$
\chi_{T}=\left(\frac{\partial \gamma}{\partial \tau}\right)_{T}
$$

The precise understanding of these divergences, and more generally of the behavior of the system near the critical point, has been one of the principal objectives of the theory of critical phenomena. Near the critical point, it is convenient to introduce the variable

$$
\theta=\frac{T-T_{c}}{T_{c}}
$$

which measures the deviation in temperature from $T_{c}$. Then one has (Yeomans, 1992)

$$
\begin{gathered}
C_{H} \sim|\theta|^{\bar{\alpha}}, \quad(H=0) \\
\gamma \sim(-\theta)^{\bar{\beta}}, \quad(H=0)
\end{gathered}
$$


Table 1 Exact critical exponents for the two-dimensional spin- $\frac{1}{2}$ Ising model and mean-field approximation

\begin{tabular}{ccccc}
\hline Exponent & $\bar{\alpha}$ & $\bar{\beta}$ & $\bar{\gamma}$ & $\bar{\delta}$ \\
\hline Ising & $0(\log )$ & $1 / 8$ & $7 / 4$ & 15 \\
Mean field & 0 & $1 / 2$ & 1 & 3 \\
\hline
\end{tabular}

$$
\begin{gathered}
\chi_{T} \sim|\theta|^{\bar{\gamma}}, \quad(H=0) \\
H \sim|\gamma|^{\bar{\delta}} \operatorname{sgn}(\gamma), \quad\left(T=T_{c}\right)
\end{gathered}
$$

for some critical exponents $\bar{\alpha}, \bar{\beta}, \bar{\gamma}$, and $\bar{\delta}$. As noted earlier, the rate of growth of the dislocation density $\rho$ also diverges at yield. It may be shown (Yeomans, 1992) that the sum of the correlation function over site pairs involved in Eq. (15) is proportional to the plastic compliance $\chi_{\tau}$. It therefore follows that the characteristic exponent for $\rho$ coincides with the characteristic exponent for $\chi_{T}$, namely $\bar{\gamma}$. The characteristic exponents obtained by an exact treatment of the Ising model and from the mean-field approximation are collected in Table 1. It should be noted that the mean-field exponents generally differ from the exact values.

Critical exponents are important because they afford a classification of critical phenomena into universality classes. They also define scaling relations between various thermodynamic quantities near the critical point. Striking evidence of universality was provided by Guggenheim (1945), who showed that the coexistence curves for eight different fluids near the critical point, when plotted in terms of reduced variables, collapse into a universal curve which can be described by a characteristic exponent. In a similar manner, whereas $T_{c}$ and other aspects of the hardening of single crystals may vary widely between materials, the characteristic exponents should be universal and therefore define material-independent scaling relations between quantities of interest, such as the critical resolved shear stress and the obstacle density.

\section{A Simple Kinetic Theory}

The equilibrium properties of the dislocation loop/obstacle system described in the foregoing rest critically on an assumption of ergodicity, i.e., the assumption that the system is free to explore the entire phase space and that all states are accessible from all other states regardless of any intervening energy barriers. Under these assumptions, the behavior of the system is reversible. Whereas instances of reversible dislocation motion exist, e.g., the "flip-flop" of Taylor lattices in fatigue crystals (KuhlmannWilsdorf, 1979), the plasticity of single crystals is most commonly observed to be irreversible and to be accompanied by hysteresis and dissipation.

The mean-field approximation developed above, in conjunction with its analogy to solid-solid phase transitions (e.g., Ericksen (1975)), affords the following simple model of hysteresis. Consider a loop/obstacle system below the critical temperature. As in the equilibrium case, the effective field $h_{0}$ is assumed to minimize the Bogoliubov function $f\left(h_{0}\right)$, Eq. (48). In the range $h<-h_{c}$ the minimizer is unique (Fig. 5, point $A$ ). Imagine now increasing the applied field $h$ monotonically above the point $h=-h_{c}$ (Fig. 5, point $B$ ). In the range $-h_{c}<h<h_{c}$, the function $f\left(h_{0}\right)$ has two minima. Of these, the leftmost point, $h_{0}<0$, is the absolute minimizer in the range $h<0$. As noted earlier, the absolute minimum shifts to the positive axis, $h_{0}>0$, for $h>0$. However, we may argue that this exchange of stability is impeded by the intervening maximum of the function $f\left(h_{0}\right)$, which plays the role of an energy barrier, and that, in consequence, the leftmost minimizer $h_{0}<0$ is the only state accessible to the system (Fig. 5, point $B$ ). In the range $h>h_{c}$ the function $f\left(h_{0}\right)$ has a unique minimizer $h_{0}>0$ (Fig. 5, point $E$ ). Therefore, it follows that $h_{0}$ must jump discontinuously at the critical point $h=h_{c}$ (Fig. 5, points $C$ and $D$ ), which therefore sets the yield point in the kinetic

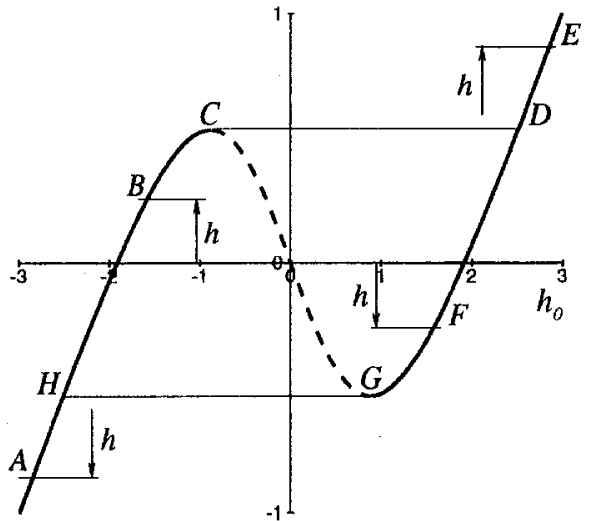

Fig. 5 Mean-fleld construction including hysteresis and plastic dissipation. Subcritical case $\kappa=2$.

theory. The corresponding critical resolved shear stress, or yield stress, then follows from (40) as

$$
t_{c}=2 \operatorname{acosh} \sqrt{\kappa}-2 \sqrt{\kappa(\kappa-1)}+\log \left(1+e^{m}\right) .
$$

This critical resolved shear stress may be rewritten directly in terms of the obstacle density $\langle\eta\rangle$ by the elimination of the chemical potential $m$ with the aid of $\mathrm{Eq}$. (52), with the result

$$
\begin{aligned}
t_{c}=2 \operatorname{acosh} \sqrt{\kappa}- & 2 \sqrt{\kappa(\kappa-1)} \\
& +\log \left(1+\frac{2\langle\eta\rangle}{1-\sqrt{1-1 / \kappa}-2\langle\eta\rangle}\right) .
\end{aligned}
$$

Imagine that the process of loading just described, in which the applied field $h$ is increased monotonically from $A$ to $E$ in Fig. 5, is followed by unloading, i.e., the applied field is subsequently decreased monotonically from $E$. The fundamental assumption is that, upon unloading the loading path $A B C D E$ is not traversed in reverse. Indeed, during unloading the point $D$ defines the absolute minimum of $f\left(h_{0}\right)$ and, therefore, the jump from $D$ to $E$ is not energetically favorable. Instead, the unloading path is postulated to be $E D G H A$, Fig. 5, and involves reverse yielding at $h=-h_{c}$. Indeed, at the yield point the effective field $h_{0}$ jumps discontinuously. The net result is a hysteresis loop $A B C D E D G H A$ and the dissipation of energy as plastic work.

It should be carefully noted that both the hysteresis model and the accessibility criteria invoked to select minimizers constitute physical postulates that are formulated in addition to the strict principles of equilibrium statistical mechanics. In particular, postulates are introduced regarding what subset of the phase space is accessible from a given state, with the result that the system is no longer ergodic. These additional postulates form the rudiments of a kinetic theory, as attested to by the irreversible and dissipative character of the predicted behavior.

\section{Summary and Concluding Remarks}

We have developed a statistical mechanical theory of forest hardening in which yielding arises naturally as a phase transition. Our focus in this paper has been to identify the simplest possible model of slip which clearly demonstrates the connection between yielding and criticality. With this objective in mind, we have focused on the motion of a single dislocation loop on a slip plane. We have assumed that the dislocations are well screened so that their energy is proportional to their length. The motion of the loop is impeded by forest dislocations piercing the slip plane, which we treat as point obstacles. The occurrence of slip at the sites occupied by these obstacles requires the expenditure of a certain amount of work commensurate with the strength of the obstacle. The case of obstacles of infinite strength has been treated in detail.

We have shown that the behavior of the dislocation loop as it 
sweeps the slip plane under the action of a resolved shear stress is identical to that of a lattice gas and, equivalently, to the twodimensional spin- $-\frac{1}{2}$ Ising model. In particular, there exists a critical temperature $T_{c}$ below which the system exhibits a yield point: The slip strain increases abruptly when the applied resolved shear stress attains a critical value. Above the critical temperature the yield point disappears and the slip strain depends continuously on the applied stress. The critical exponents, which describe the behavior of the system near the critical temperature, coincide with those of the two-dimensional spin- $\frac{1}{2}$ Ising model.

It is revealing to compute the transition temperature $T_{c}$ for specific materials. For the sake of argument let us simply assume that the shear modulus decreases linearly with temperature and vanishes at the melting temperature $T_{m}$, i.e.,

$$
G=G_{0}\left(1-\frac{T}{T_{m}}\right)
$$

where $G_{0}$ is the shear modulus at zero temperature. Inserting this relation into the expression (54) for the critical temperature yields

$$
T_{c}=\left(\frac{1}{T_{0}}+\frac{1}{T_{m}}\right)^{-1}
$$

where

$$
T_{0}=K_{c}^{-1} \frac{C G_{0} b^{2} a}{2 k}
$$

Taking copper by way of example, one has $G_{0} \approx 40 \mathrm{GPa}, b=$ $2.556 \AA, a \approx b, k=1.380 \times 10^{-23} \mathrm{~J} \mathrm{~K}^{-1}, C \approx 0.3$, and $T_{m}=$ $1,343 \mathrm{~K}$. With these constants, $(73)$ gives $T_{0}=16,474 \mathrm{~K}$ and (72) $T_{c}=1,241 \mathrm{~K}$, or 92 percent of the melting temperature. This example suggests that metals remain within the subcritical regime and, consequently, exhibit a yield point, up to temperatures very close to the melting temperature, although the sharpness of yielding should be blunted with increasing temperature. These general conclusions are indeed in keeping with observation.

Unfortunately, the full effect of temperature on the behavior of the system cannot be ascertained without knowing the dependence of the elastic moduli on temperature. Thus, linear elastic estimates predict the line tension, Eq. (1), and the obstacle strength, Eq. (4), to be proportional to the shear modulus $G$, which is itself a strong function of temperature. Under these conditions, as the crystal approaches melting $G$ tends to zero and, correspondingly, both the line tension and the obstacle strength should decrease to zero. This in turn accounts for the thermal softening observed to occur in many ductile crystals with increasing temperature.

As stated above, the system defined by a single dislocation loop moving through forest dislocations has the virtue of exhibiting the connection between criticality and yielding in the simplest possible terms. However, in this model the slip displacement is restricted to be either 0 or $b$ and the maximum slip strain which can be born by a slip system is (36). By way of contrast, the unconstrained plastic flow characteristic of the macroscopic behavior of single crystals requires the simultaneous operation of many dislocation loops on each slip plane. An extension of the Hamiltonian (7) which accounts for this effect may be obtained by allowing the slip $\xi_{i}$ at site $i$ to take any nonnegative integer value. The resulting Hamiltonian, however, cannot be solved analytically but should yield to approximation techniques such are mean-field theory based, e.g., on a mean-field energy of the form (44).

Also in the interest of simplicity, we have given special attention to obstacles of infinite strength. The treatment of obstacles of finite strength does not offer any particular difficulties. A more challenging extension concerns the combined hardening effect of obstacles of different strengths, e.g., such as are introduced by the activation of more than one secondary system. Foreman and Makin (Foreman, 1955; Foreman and Makin, 1966) have investigated this problem numerically, and have characterized the effective strength of the obstacles. Here again, an extension of the Hamiltonian (7) which accounts for more than one obstacle species is

$$
\begin{aligned}
E=\sum_{\langle i, j\rangle} \frac{\Gamma a}{2}\left(\xi_{i}-\xi_{j}\right)^{2}-\tau b a^{2} \sum_{i=1}^{N} \xi_{i} \\
+\sum_{s=1}^{s}\left\{f^{(s)} a \sum_{i=1}^{N} \xi_{i} \eta_{i}^{(s)}-\mu^{(s)} \sum_{i=1}^{N} \eta_{i}^{(s)}\right\} .
\end{aligned}
$$

Here the sum over $s$ extends to the $S$ species of forest dislocations contributed by different secondary systems; the variable $\eta_{i}^{(s)}$ is 1 if site $i$ is occupied by an obstacle of type $s$ and is 0 otherwise; $f^{(s)}$ is the strength of the obstacles of type $s$; and $\mu^{(s)}$ is a chemical potential to be determined on the condition that the density of obstacles of type $s$ match a prescribed value. It should be noted that, in this model, a site $i$ may be occupied by several obstacles of different species. As in the case $S=1$ considered in this paper, Eq. (16), the sums over the obstacle occupancy fields $\eta_{i}^{(s)}$ are trivial. The resulting model should provide a precise characterization of how obstacles of different strengths cooperate to determine the hardening characteristics of a crystal.

Other worthwhile extensions of the basic Hamiltonian (7) might account for the energy required to activate dislocation sources, e.g., of the Frank-Read type; the Peierls stress required to overcome the resistance of the atomic lattice to dislocation slip, specially in materials other than elemental metals where such barrier is not negligible; the inertia attendant to the motion of dislocations; the anisotropy of the elastic moduli and of the line tension; and other effects. Evidently, while these extensions enhance the predictive character of the theory, they are also introduced at a certain expense as regards the complexity of the theory. In any case, the statistical mechanical framework sketched out in this paper may open the way for a physics-based description of crystalline slip which benefits from the tools and principles of the theory of critical phenomena.

\section{Acknowledgments}

The support of the DOE through Caltech's ASCI Center for the Simulation of the Dynamic Response of Materials is gratefully acknowledged.

\section{References}

Abeyaratne, R., and Knowles, J. K., 1988, "On the dissipative response due to discontinuous strains in bars of unstable elastic material," International Journal of Solids and Structures, Vol. 24, No. 10, pp. 1021-1044.

Baskes, M. I., Hoagland, R. G., and Tsuji, T., 1997, "An atomistic study of the strength of an extended dislocation barrier."

Baxter, R. J., Exactly Solved Models in Statistical Mechanics, Academic Press, London.

Binder, K., ed., 1986, Monte Carlo Methods in Statistical Physics, 2nd Ed., Springer-Verlag, New York.

Binder, K., ed., 1987, Applications of the Monte Carlo Method in Statistical Physics, 2nd Ed., Springer-Verlag, New York.

Binney, J. J., Dowrick, N. J., Fisher, A. J., and Newman, M. E. J., 1992, The Modern Theory of Critical Phenomena, Clarendon Press, Oxford.

Chaikin, P. M., and Lubensky, T. C., 1995, Principles of condensed matter physics, Cambridge University Press, Cambridge, UK.

Chrzan, D. C., and Mills, M. J., 1993, "Collective behavior and superdislocation motion in $1 \mathrm{H}_{2}$ alloys," Materials Science and Engineering $A$, Vol. 164, No. 1-2, pp $82-92$.

Chrzan, D. C., and Mills, M. J., 1994, "Criticality in the plastic deformation of 11 intermetallic compounds," Physical Review B, Vol. 50, No. I, pp. 30-42.

Cuitiño, A. M., 1996, "Effect of temperature and stacking fault energy on the hardening of fec crystals," Materials Science and Engineering, Vol. A216, pp. $104-116$.

Cuitiño, A. M., and Ortiz, M., 1992, "Computational Modelling of Single Crystals," Modelling and Simulation in Materials Science and Engineering, Vol. 1, pp. 255-263.

Cuitiño, A. M., and Ortiz, M., 1993, "Constitutive Modeling of $\mathrm{LL}_{2}$ Intermetallic Crystals," Materials Science and Engineering, Vol. A170, pp. 111-123.

Ericksen, J. L., 1975, "Equilibrium of bars," Journal of Elasticity, Vol. 5, pp. 191-201.

Feynman, R. P., 1972, Statistical Mechanics, Addison-Wesley, Reading, MA. 
Foreman, A. J., 1995, "Dislocation energies in anisotropic crystals," Acta Metallurgica, Vol. 3, pp. 322-330.

Foreman, A. J. E., and Makin, M. J., 1966, "Dislocation Movement through Random Arrays of Obstacles," Philosophical Magazine, Vol. 14, p. 911.

Foreman, A. J. E., and Makin, M. J., 1967, "Dislocation movement through random arrays of obstacles," Canadian J. Phys., Vol. 45, p. 273.

Grosskreutz, J. C., and Mughrabi, H., 1975, "Description of the work-hardened structure at low temperature in cyclic deformation," Constitutive Equations in Plasticity, A. S. Argon, ed., M.I.T. Press, Cambridge, MA, pp. 251-326.

Guggenheim, E. A., 1945, "The Principle of Corresponding States" Journal of Chemical Physics, Vol. 13, pp. 253-261.

Hansen, N., and Kuhlmann-Wilsdorff, D., 1986, "Low Energy Dislocation Structures due to Unidirectional Deformation at Low Temperatures," Materials Science and Engineering, Vol. 81, pp. 141-161.

Hirth, J. P., and Lothe, J., 1968, Theory of Dislocations, McGraw-Hill, New York James, R. D., 1979, "Co-exitent phases in the one-dimensional theory of elastic bars," Archive for Rational Mechanics and Analysis, Vol. 72, pp. 99-140.

Kocks, U. F., 1964, "Latent hardening and secondary slip in aluminum and silver," Transactions of the Metallurgical Society of the AIME, Vol. 230, p. 1160.

Kocks, U. F., Franciosi, P., and Kawai, M., 1991, "A forest model of latent hardening and its applications to polycrystal deformation," Textures and Microstructures, Vol. 14-18, pp. 1103-1114.

Koonin, S. E., and Meredith, D. C., 1990, Computational Physics, AddisonWesley, Reading, MA.

Kosterlitz, J. M., and Thouless, D. J., 1972, "Long Range Order and Metastability in Two Dimensional Solids and Superfluids," Journal of Physics C, Vol. 5, pp. L124-L126.

Kosterlitz, J. M., and Thouless, D. J., 1973, "Ordering, Metastability and Phase Transitions in Two-Dimensional Systems" Journal of Physics C, Vol. 6, pp. 1181-1203.
Kovacs, I, 1967, "The Mechanism of the Work-Harderning in F.C.C. Metals," Acta Metallurgica, pp. 1731-1736.

Kovács, I., and Zsoldos, L., 1973, Dislocation and plastic deformation, Pergamon

Press, Oxford, UK

Kuhlmann-Wilsdorf, D., 1979, "Dislocation behavior in fatigue. IV. Quantitative interpretation of friction stress and back stress derived from hysteresis loops," Materials Science and Engineering, Vol. 39, pp. 231-245.

Kuhlmann-Wilsdorf, D., 1989, "Theory of plastic deformation: properties of low energy dislocation structures," Materials Science and Engineering, Vol.

A113, p. 1 .

Mughrabi, H., 1975, "Description of the Dislocation Structure after Unidirectional Deformation at Low Temperatures," Constitutive Equations in Plasticity, A. S. Argon, ed., M.I.T. Press, Cambridge, MA, pp. 199-250.

Nabarro, F. R. N., 1967, Theory of crystal dislocations, Oxford University Press, Oxford, UK.

Nelson, D. R., and Halperin, B. I., 1979, "Dislocation-Mediated Melting in Two Dimensions," Physical Review B, Vol. 19, pp. 2457-2484.

Ortiz, M, and Popov, E. P., 1962, "A Statistical Theory of Polycrystalline Plasticity," Proceedings of the Royal Society of London, Vol. A379, pp. 439-458.

Ortiz, M., and Repetto, E. A., 1998, "Nonconvex energy minimization and dislocation structures in ductile single crystals," Journal of the Mechanics and Physics of Solids, in press.

Phillips, R., and Shenoy, V., 1998, manuscript in preparation.

Stanley, H. E., 1971, Introduction to Phase Transitions and Critical Phenomena, Oxford University Press, Oxford, UK.

Yeomans, J. M., 1992, Statistical Mechanics of Phase Transitions, Clarendon Press, Oxford, UK.

Young, A. P., 1979, "Melting and the Vector Coulomb Gas in two Directions," Physical Review B, Vol. 19, pp. 1855-1866. 